\title{
Abundance Distributions in Barred Galaxies
}

\author{
Jean-René Roy \\ Département de Physique and Observatoire du Mont Mégantic, \\ Université Laval, Québec, Qc G1K 7P4, Canada
}

\begin{abstract}
I review the evidence for global abundance gradients in barred galaxies to be flatter when compared with those of normal galaxies. The finding that the abundance gradient becomes flatter as the strength of the bar increases, illustrates the effectiveness of radial flows induced by bars at transporting large amount of gas across galaxy disks.
\end{abstract}

\section{Introduction}

Bars are a powerful and efficient means to transport large quantities of gas across galaxy disks over a period shorter than a Hubble time (Sellwood \& Wilkinson 1993; Friedli \& Benz 1993). This can modify the star formation activity across the disk; thus one can expect different chemical enrichment histories when comparing galaxies with bars with those without (Edmunds \& Greenhow 1995). How do abundance distributions in barred galaxies differ from those of normal galaxies?

Because the abundance levels found in large disk galaxies are moderately high, the derivation of abundances is achieved by employing diagnostic nebular

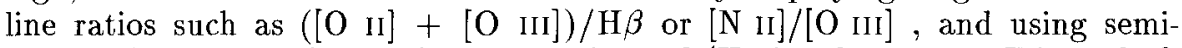
empirical schemes relating their strengths to $\mathrm{O} / \mathrm{H}$ abundances; see Edmunds \& Pagel (1984), McCall et al. (1985), Dopita \& Evans (1986), Edmunds (1989), or McGaugh (1991). Radial gradients in these line ratios reflect primarily abundance gradients. The absolute $\mathrm{O} / \mathrm{H}$ abundance predicted by these calibrations can vary whether one is using one or the other calibrator, but the relative trends can be probed reliably by either one, as shown by the close relationship between $\left(\left[\mathrm{O}_{\mathrm{II}}\right]+\left[\mathrm{O}_{\mathrm{III}}\right]\right) / \mathrm{H} \beta$ and $[\mathrm{N} \mathrm{II}] /\left[\mathrm{O}_{\mathrm{III}}\right]$ in Ryder (1995).

Abundance indicators must be used with care. Factors other than heavy element abundances also modify nebular line strengths: The ionization parameter and the mean ionizing temperature of the radiation field affect the line ratios resulting in uncertainties of $\leq \pm 0.2$ dex in the abundance derived from individual $\mathrm{H}$ II regions. The uncertainties tend to be higher at abundances $\geq O / H_{\odot}$ (Henry 1993; Oey \& Kennicutt 1993). We assume that these uncertainties do not conspire significantly with properties like bars or other parameters which would be a changing function of radius. To study differences between barred and non-barred galaxies, it is necessary to compare gradients derived from the same calibration.

The abundances are those of the ionized interstellar medium, and are restricted mostly to galaxies of high surface brightness and of late types ( $\mathrm{SBc}$, 
SBb). Non-barred large-disk galaxies, e.g. NGC 628, M 33 and NGC 2403, have strong global O/H gradients of similar amplitude (Belley \& Roy 1992; Zaritsky et al. 1994): $\sim-0.09 \mathrm{dex} / \mathrm{kpc}$ or $\sim-0.8 \mathrm{dex} / \rho_{0}$, where $\rho_{0}$ is the normalized isophotal radius, and where we use the $\mathrm{O} / \mathrm{H}$ calibration of Edmunds \& Pagel; Using the calibration of Dopita \& Evans (1986) of the same line ratio would give shallower gradients by about a factor of two (e.g. Walsh \& Roy 1989).

Finally, abundance gradients are defined in relation to a normalizing radius; there are uncertainties associated with the normalizing radius whether the slopes are expressed in $\operatorname{dex} / \mathrm{kpc}, \operatorname{dex} / R_{25}$, or $\operatorname{dex} / \rho_{0}$. I refer the reader to the papers of Vila-Costas \& Edmunds (1992) and Zaritsky et al. (1994) for a full discussion of this issue.

\section{The Prehistory - Before 1981}

Pagel \& Edmunds (1981) reviewed early studies. Their conclusion of possibly flatter gradients in galaxies with a bar was based primarily on the spectrophotometry of the spectacular barred spiral galaxy NGC 1365 by Alloin et al. (1981): These authors asserted that "If we permit ourselves, to make the bold assumption that NGC 1566 has a typical abundance distribution for $S b$-Sc galaxies, then the data [for NGC 1365] could be taken as suggesting that an abundance gradient along the bar is either smoothed out or inhibited from coming about by non-circular streaming motions."

This was also consistent with the result found by Pagel et al. (1978) for the Magellanic Clouds: "In the SMC, there is no sign of any gradient, which suggests that the entire $S M C$ is well mixed, probably owing to the streaming or circulating mass motions that may be associated with the presence of a bar; one could imagine that a similar process has been operating to reduce the abundance gradient in the $L M C$." It is interesting to see that even with the meager sampling of the above galaxies, the early conclusion for flatter gradients in barred galaxies was essentially correct.

\section{The Middle Age - 1981 to 1990}

During the 1980's, improving detectors and spectrophotometric techniques, and nebular modeling were the major lines of work in chemical research of galaxies. Although little attention was given to the chemistry of barred galaxies, major new understanding was achieved leading to the surface density/abundance relation, to a better knowledge of the yields in various galaxies, and of relative abundance of elements vs. nucleosynthesis and chemical evolution scenarios.

Most of the results were gathered, analyzed and discussed in the review by Vila-Costas \& Edmunds (1992). They identified the distinct behavior of barred galaxies: "It is apparent that the barred galaxies have only very small gradients (although we accept that our sample - five galaxies - is small). The gradients for non-barred galaxies are steeper, and there is a correlation of gradient with morphological type, the later types having steeper gradients." 


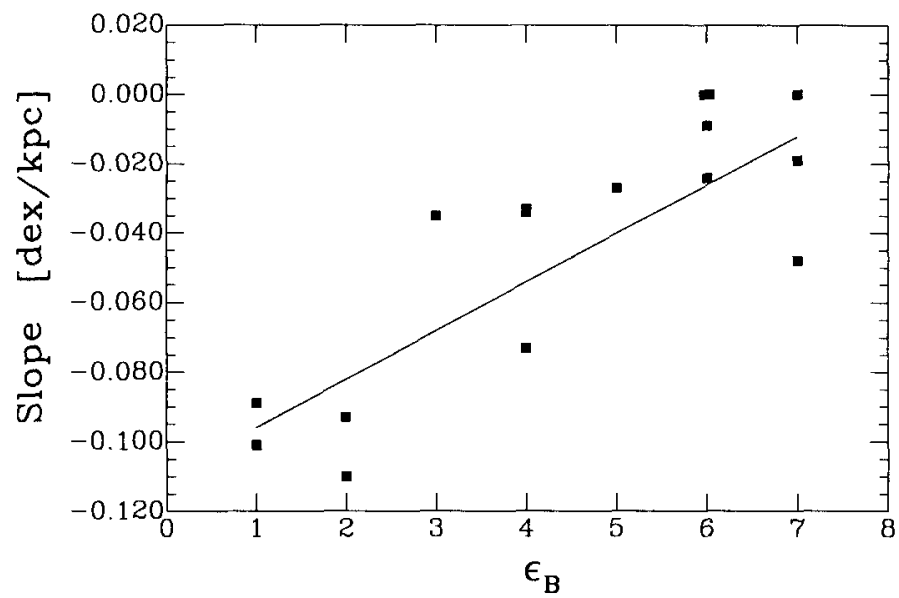

Figure 1. Slope of $\mathrm{O} / \mathrm{H}$ abundance gradients in barred galaxies as a function of the stellar bar axis ratio following Martin \& Roy (1994).

\section{The Enlightenment - 1990 to Present}

Our gains in nebular physics rapidly gave tangible results through the new technologies. The late 1980's and the 1990's saw the introduction of multi-object spectroscopy and of spectrophotometric imaging with narrow-band filters; these techniques combined with detectors of increased efficiency gave the large samples of $\mathrm{H}$ II regions that allowed to reveal differences between the abundance distributions in barred and "normal" galaxies. Still the overall number of wellsampled galaxies remains small, i.e. $\leq 40$ normal/barred galaxies mainly of Sbc types.

From a systematic study of abundances in 39 galaxies, Zaritsky et al. (1994) conld conclude on galaxies with bars: "Overall, we find a strong indication that barred galaxies do have flatter gradients than unbarred galaxies. However, a few unbarred galaxies have gradients that are significantly shallower than the average, and some barred galaxies have been shown to have normal gradients (NGC 4303)." The last sentence points out that the slopes of abundance global gradient may vary among barred galaxies.

The overall behavior was clarified by Martin (1992) and Martin \& Roy (1994) who completed the first thorough study of $\mathrm{O} / \mathrm{H}$ abundances in barred galaxies. They confirmed that global $\mathrm{O} / \mathrm{H}$ gradients in intermediate and barred galaxies are shallower than gradients in normal galaxies. In addition they found that the $\mathrm{O} / \mathrm{H}$ gradients become flatter as the relative strength of the bar increases (Figure 1). To emphasize this effect, they proposed to relate the slope of the abundance gradient with the bar ellipticity, $\epsilon_{B}$, defined as

$$
\epsilon_{B}=10(1-\mathrm{b} / \mathrm{a})
$$


where $b / a \equiv$ stellar bar axis ratio; the bar strength increases with $\epsilon_{B}$ (see Martin 1995). The trend of Figure 1 is quantified by the following relation:

$$
\Delta \log \mathrm{O} / \mathrm{H} / \Delta \mathrm{R}(\operatorname{dex} / \mathrm{kpc}) \approx-0.110+0.014 \epsilon_{B}
$$

The $O / H$ gradients become flatter as the bar ellipticity, $\epsilon_{B}$, increases. This trend between the slope of the $\mathrm{O} / \mathrm{H}$ radial gradient and the strength of the bar has been modeled successfully by Friedli \& Benz (1995).

The above finding applies to large disk galaxies. Does the trend continue with low mass galaxies where, like in the Large Magellanic Cloud, a bar is often a dominant optical structure? Edmunds \& Roy (1993) have noted that steep abundance gradients in gas-rich disk galaxies require the presence of unbarred spiral structure: Abundance gradients disappear at the same absolute magnitude that spiral structure ceases. This suggests that the radial abundance distributions in small disk galaxies are flat. Indeed small galaxies like NGC 1569, NGC 2366, and NGC 4395 look like the MC's; their radial abundance distribution appears flat within the uncertainties and the intrinsic dispersion (Roy et al. 1995).

\section{The Puzzles}

There are however exceptions to the general behavior described above.

\section{A) The Case of NGC 4303}

The first anomaly is presented by the Virgo spiral NGC 4303. This barred galaxy has a near-normal global abundance gradient of $-0.07 \mathrm{dex} / \mathrm{kpc}$ (Henry et al. 1992; Martin \& Roy 1992). The bar is moderately strong $\left(\epsilon_{B}=4\right)$ and a flatter gradient would be expected. The galaxy is known to have an abnormally high star formation rate, which may be linked to a recent tidal interaction with two nearby companions NGC 4292 and NGC 4303A (Cayatte et al. 1990). Is the high star formation and heavy element enrichment rate in NGC 4303 compensating for the dilution effect of the bar?

\section{B) The "Breaks" in NGC 3319 and NGC 3359}

NGC 3319 was observed by Zaritsky et al. (1994) and NGC 3359 (Figure 2) by Martin \& Roy (1995). These two barred galaxies present strong and obvious breaks in their radial $\mathrm{O} / \mathrm{H}$ distributions. In NGC 3359, the break occurs just beyond the co-rotation radius (Figure 3 ). Although puzzling, the observed break is entirely consistent with the model of secular evolution of barred galaxies by Friedli et al. (1994) and Friedli \& Benz (1995) (Figure 3). Such breaks are expected in galaxies with bars in the early phase of formation; during that phase, inward radial flows generated by the bar can sustain strong star formation and a steep inner abundance gradient, while beyond co-rotation, the outward radial flow is most efficient at diluting and mixing gas radially, producing there a flat abundance gradient.

Finally if one assumes that the large scatter of $\mathrm{O} / \mathrm{H}$ at a given radius is reflecting local enrichment in a timescale shorter than the azimuthal and radial 

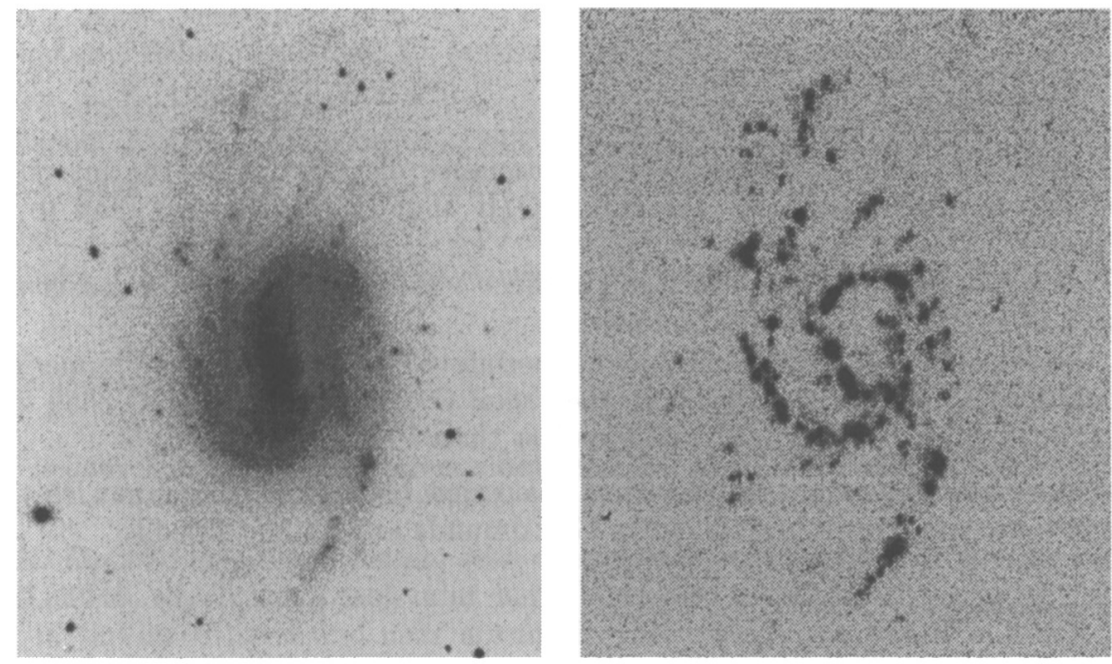

Figure 2. Red continuum image $(7020 \AA, \Delta \lambda=200 \AA)$ of the barred galaxy NGC 3359 at left, and at right, the same galaxy in $\mathrm{H} \alpha$ (from Martin \& Roy 1995).
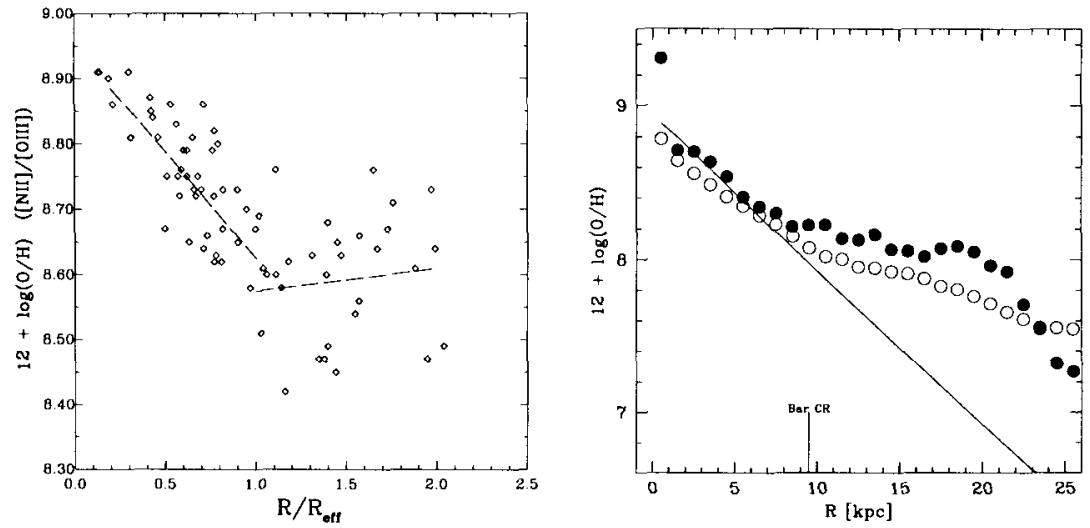

Figure 3. At left are the observed $\mathrm{O} / \mathrm{H}$ abundances across NGC 3359 derived with the $[\mathrm{N} \mathrm{II}] /[\mathrm{O}$ III] indicator from Martin \& Roy (1995). This is compared at right with the results of numerical simulations showing gaseous (solid circles) and stellar (open circles) radial abundance gradients 1 Gyr after a bar has formed (Friedli et al. 1994). 
mixing timescales, an age of $\sim 400 \mathrm{Myr}$ is derived for the bar in NGC 3359 (Roy \& Kunth 1995; Martin \& Roy 1995).

\section{Summary}

The presence of a bar influences the abundance distribution in the interstellar medium of disk galaxies: the global $\mathrm{O} / \mathrm{H}$ abundance gradients tend to be shallower.

$\diamond$ This is best illustrated by the "Martin effect": the abundance gradient becomes flatter when the bar ellipticity increases.

$\diamond \ldots$ and the influence of the bar may be modulated by its age. Indeed numerical simulations show that the breaks in the radial $\mathrm{O} / \mathrm{H}$ gradient in NGC 3359 and NGC 3319 can be the signature of bars less than $500 \mathrm{Myr}$ old.

Acknowledgments. In preparing this review I benefited from many fruitful discussions with Pierre Martin, Mike G. Edmunds and Daniel Friedli.

\section{References}

Alloin, D., Edmunds, M. G., Lindblad, P. O., \& Pagel, B. E. J. 1981, A\&A, 101, 377

Belley, J. \& Roy, J.-R. 1992, ApJS, 87, 61

Cayatte, V., van Gorkom, J. H., Balkowski, C., \& Kotanyi, C. 1990, AJ, 100, 604

Dopita, M. A. \& Evans, I. N. 1986, ApJ, 307, 431

Edmunds, M. G. 1989, in Evolutionary Phenomena in Galaxies, J. E. Beckman \& B. E. J. Pagel, Cambridge University Press, 356

Edmunds, M. G. \& Pagel, B. E. J. 1984, MNRAS, 211, 507

Edmunds, M. G. \& Roy, J.-R. 1993, MNRAS, 261, L17

Edmunds, M. G. \& Greenhow, R. M. 1995, MNRAS, 272, 241

Friedli, D. \& Benz, W. 1993, A\&A, 268, 65

Friedli, D., Benz, W., \& Kennicutt, R. C. 1994, ApJ, 430, L105

Henry, R. B. C. 1993, MNRAS, 261, 306

Henry, R. B. C., Pagel, B. E. J., Lasseter, D. F., \& Chincarini, G. L. 1992, MNRAS, 258, 321

Martin, P. 1992, Ph. D. thesis, Université Laval

Martin, P. 1995, AJ, 109, 2428

Martin, P. \& Roy, J.-R. 1992, ApJ, 397, 463

Martin, P. \& Roy, J.-R. 1994, ApJ, 424, 599

Martin, P. \& Roy, J.-R. 1995, ApJ, 445, 161

McGaugh, S. S. 1991, ApJ, 380, 140

Oey, M. S. \& Kennicutt, R. C. 1993, ApJ, 411, 137

Pagel, B. E. J., Edmunds, M. G., Fosbury, R. A. E., \& Webster, B. L. 1978, MNRAS, 184,569

Pagel, B. E. J. \& Edmunds, M. G. 1981, ARAA, 19, 77 
Roy, J.-R. \& Belley, J. 1993, ApJ, 406, 60

Roy, J.-R. \& Kunth, D. 1995, A\&A, 294, 432

Roy, J.-R., Belley, J., Dutil, Y., \& Martin, P. 1995, ApJ, submitted

Ryder, S. D. 1995, ApJ, 444, 610

Sellwood, J. A. \& Wilkinson, A. 1993, Rep. Prog. Phys., 56, 173

Vila-Costas, M. B. \& Edmunds, M. G. 1992, MNRAS, 259, 121

Walsh, J. R. \& Roy, J. R. 1989, ApJ, 341, 722

Zaritsky, D., Kennicutt, R. C., \& Huchra, J. P. 1994, ApJ, 420, 87

\section{Discussion}

M. Regan: Do you see a difference between the abundance gradient within the radius of the bar and at radii greater than the length of the bar?

J.-R. Roy: So far differences are seen clearly only in NGC 3319 and NGC 3359. NGC 6946, which has a molecular bar, shows also a break in its gradient; it is flat in the inner parts and near normal in the outer parts, but it is a difficult and probably marginal case (see Roy \& Belley 1993).

S. Ryder: Have you searched for any correlation of the radial abundance gradient profiles with radial surface brightness profiles, or with kinematics?

J.-R. Roy: No, we have not. This is a step to be taken to establish whether the abundance vs. surface density relation is different.

J. A. Sellwood: Two concerns and a question: First, the difference between abundance gradients in unbarred and barred galaxies would seem to be a strong argument against the idea of recurring bars. Second, non-circular motions induced by a bar or other asymmetry mean that gas streams on elliptical and not circular orbits. Therefore, the instantaneous position of the $\mathrm{H}$ II region could be a kpc or more away from the "home radius" of that parcel of gas. Is there any way you could develop estimates of the radial distances of $\mathrm{H}$ II regions that more closely reflect the home radius of the measured gas?

J.-R. Roy: Ouf!

J. Beckman: Would it not be valuable to confirm (or otherwise) your measurements of the morphology-abundance gradient relation by measuring more than one "metal"? If you found flat gradients in two species, this would surely be powerful support for your present conclusions.

J.-R. Roy: In general abundances of elements other than oxygen are less reliable because we cannot yet observe all their main ionization stages. However with the new near-infrared and infrared techniques, it will become possible to derive the abundance distributions of nitrogen, sulfur, argon and others in several galaxies.

Z. Tsvetanov: Do you have any indication whether NGC 4303 is a product of a merger?

$J .-R$. Roy: Apart from possible tidal interaction with two companions, there is no clear evidence of a recent merger. 